\title{
Characterization of the Plastid RNA Polymerase Complex
}

\author{
Sangyool Lee ${ }^{1}$, Sun Hyun Chang ${ }^{1}$, Taeyoung Um¹, Geupil Jang ${ }^{1}$, Ju-Kon Kim², Yang Do Choi ${ }^{1}$ \\ ${ }^{1}$ Department of Agricultural Biotechnology and Research Institute of Agriculture and Life Sciences \\ Seoul National University \\ Seoul 08826, Korea \\ leesangyool@snu.ac.kr; dacadaca@snu.ac.kr; tyum@snu.ac.kr; yk3@snu.ac.kr; choiyngd@snu.ac.kr \\ ${ }^{2}$ Graduate School of International Agricultural Technology and Crop Biotechnology Institute \\ Green BioScience and Technology, Seoul National University \\ Pyeongchang 25354, Korea \\ jukon@snu.ac.kr
}

\section{Extended Abstract}

Plant growth and productivity largely rely on the chloroplast biogenesis, which are responsible for photosynthesis and the biosynthesis of amino acids and phytohormones. Chloroplasts were symbiotically acquired by heterotrophic unicellular eukaryotes and massive transfer of plastid genes to the host nucleus during evolution transformed plastids into semiautonomous organelles. The chloroplast genome of land plants contains genes related to photosynthesis, and plastidencoded RNA polymerase (PEP) plays a key role in expression of the photosynthetic genes and formation of fully active chloroplasts. Therefore, it is important to understand the mechanism underlying the regulation of PEP activity for creating the transgenic crops with high-yield trait. Previous genetic approaches have demonstrated that the PEP activity largely depends on at least 12 PEP-associated proteins (PAPs) by showing that PAP mutant plants exhibit suppression of plastid gene expression, resulting in defects in chloroplast biogenesis and leaf greening. Recent studies reported that PAP proteins extensively interact with other PAPs or PEP core enzymes to form PEP-complex, suggesting that assembly of PEPcomplex through the protein-protein interaction is a key mechanism controlling PEP activity and chloroplast gene expression [1].

Here, we identified the Arabidopsis thaliana seedling lethal mutant. Transmission electron microscopy showed that the chloroplasts of the mutants were poorly organized without proper stacks of thylakoid membranes while chloroplasts of wild-type plants had well-organized thylakoid membranes, indicating that this mutant has severe defects in chloroplast biogenesis. Through genotyping and expression analysis, we found that the mutant phenotype is caused by the suppression of PLASTID S1 RNA-BINDING DOMAIN PROTEIN (pTAC10/PAP3). To understand pTAC10 function in PEP-complex formation, we isolated 12 genes encoding PAPs or PEP core enzymes, and analyzed the interactions of the encoded proteins with pTAC10 through yeast two-hybrid and GST pull-down assay. From these approaches, we found that pTAC10 has the ability to interact with other PAPs, such as FSD2, FSD3, TrxZ, pTAC7, and pTAC14, but it does not interact with PEP core enzymes, such as rpoA and rpoB. Further analysis of pTAC10 interactions using a series of truncated pTAC10 proteins revealed that the truncated pTAC10 proteins lacking the C-terminal region downstream of the S1 RNA-binding domain does not interact with the PAPs such as FSD2, FSD3, TrxZ, pTAC7, and pTAC14 unlike intact pTAC10 protein. More importantly, transgenic ptac10-1 plants in which the truncated pTAC10 cDNA was overexpressed under the control of $35 \mathrm{~S}$ promoter had defects in chloroplast development, indicating that that truncated pTAC10 protein is not sufficient for recovering the ptac10-1 mutant phenotype. Collectively, these suggest that pTAC10 is an essential PAP for the establishment of PEP complex, and its C-terminal region plays a pivotal role in this process by meditating the pTAC10 interaction with other PAP proteins.

\section{References}

[1] J. Pfalz and T. Pfannschmidt, "Essential nucleoid proteins in early chloroplast development," Trends in Plant Science, vol. 18, no. 4, pp. 186-194, 2013. 